\title{
Protective effect of biodegradable nerve conduit against peripheral nerve adhesion after neurolysis
}

\author{
Kosuke Shintani, MD, ${ }^{1}$ Takuya Uemura, MD, PhD, ${ }^{1}$ Kiyohito Takamatsu, MD, PhD, ${ }^{2}$ \\ Takuya Yokoi, MD, ${ }^{1}$ Ema Onode, MD, ${ }^{1}$ Mitsuhiro Okada, MD, PhD, ${ }^{1}$ and \\ Hiroaki Nakamura, MD, PhD' \\ 1Department of Orthopaedic Surgery, Osaka City University Graduate School of Medicine; and 2Department of Orthopaedic \\ Surgery, Yodogawa Christian Hospital, Osaka, Japan
}

OBJECTIVE Peripheral nerve adhesion caused by extraneural and intraneural scar formation after neurolysis leads to nerve dysfunction. The authors previously developed a novel very flexible biodegradable nerve conduit composed of poly(L-lactide) and poly(ع-caprolactone) for use in peripheral nerve regeneration. In the present study, they investigated the effect of protective nerve wrapping on preventing adhesion in a rat sciatic nerve adhesion model.

METHODS Rat sciatic nerves were randomly assigned to one of the following four groups: a no-adhesion group, which involved neurolysis alone without an adhesion procedure; an adhesion group, in which the adhesion procedure was performed after neurolysis, but no treatment was subsequently administered; a nerve wrap group, in which the adhesion procedure was performed after neurolysis and protective nerve wrapping was then performed with the nerve conduit; and a hyaluronic acid $(\mathrm{HA})$ group, in which the adhesion procedure was performed after neurolysis and nerve wrapping was then performed with a $1 \%$ sodium HA viscous solution. Six weeks postoperatively, the authors evaluated the extent of scar formation using adhesion scores and biomechanical and histological examinations and assessed nerve function with electrophysiological examination and gastrocnemius muscle weight measurement.

RESULTS In the adhesion group, prominent scar tissue surrounded the nerve and strongly adhered to the nerve biomechanically and histologically. The motor nerve conduction velocity and gastrocnemius muscle weight were the lowest in this group. Conversely, the adhesion scores were significantly lower, motor nerve conduction velocity was significantly higher, and gastrocnemius muscle weight was significantly higher in the nerve wrap group than in the adhesion group. Additionally, the biomechanical breaking strength was significantly lower in the nerve wrap group than in the adhesion group and HA group. The morphological properties of axons in the nerve wrap group were preserved. Intraneural macrophage invasion, as assessed by the number of CD68- and CCR7-positive cells, was less severe in the nerve wrap group than in the adhesion group.

CONCLUSIONS The nerve conduit prevented post-neurolysis peripheral nerves from developing adhesion and allowed them to maintain their nerve function because it effectively blocked scarring and prevented adhesion-related damage in the peripheral nerves.

https://thejns.org/doi/abs/10.3171/2017.4.JNS162522

KEY WORDS nerve conduit; peripheral nerve; adhesion; scar; nerve wrapping; rat

$\mathrm{N}$ EUROLYSIS of peripheral nerves is an effective and frequently performed surgical procedure for entrapment neuropathy. However, postoperative perineural scarring and adhesion often cause secondary neuropathy. ${ }^{44,52}$ Once peripheral nerves are injured by trauma or neurolysis, scar tissue with infiltration of fibroblasts and myofibroblasts develops and peripheral nerve adhesion is completed. If the peripheral nerves are compressed or tethered by the scar tissue and adhesion, they become dysfunctional. ${ }^{1,732,35,36}$ Extraneural scarring also suppresses

ABBREVIATIONS HA = hyaluronic acid; PCA = poly( $\varepsilon$-caprolactone); PLA = poly(L-lactide $)$

SUBMITTED October 10, 2016. ACCEPTED April 14, 2017.

INCLUDE WHEN CITING Published online October 20, 2017; DOI: 10.3171/2017.4.JNS162522. 
blood flow affecting the peripheral nerves and thickens the epineurium and perineurium, leading to intraneural scarring that further suppresses the blood flow. $1,7,35,44,65$

For neurolysis-treated peripheral nerves, various procedures that involve wrapping veins or free or pedicled flaps with muscle or adipose tissue have been reported to prevent adhesion. . $^{12,14,16,34,38,45,47,50,58,62}$ However, because these methods involve the use of autologous normal tissues, they have major drawbacks, including donor-site morbidity and a high risk of surgical complications. Many nerve protective materials including silicone sheets, collagen tubes, gels, and fluids have been developed in recent years to overcome these drawbacks. ${ }^{2,3,6,9,13,15,19-21,23-26,29,31,39-41,45,46,60,64}$

We previously developed a novel biodegradable nerve conduit composed of poly(L-lactide) (PLA) and poly( $\varepsilon$ caprolactone) (PCL) to treat peripheral nerve injury and confirmed that axonal regeneration was induced within the conduit. ${ }^{22,30,40,56,57}$ This nerve conduit comprises 2 layers: an outer layer composed of PLA multifilament fiber mesh and an inner layer composed of a PLA- and PCL-containing porous sponge with pores of 10 to $50 \mu \mathrm{m}$. It is soft and flexible enough to cover the peripheral nerve with minimal damage, and it is degraded and absorbed very slowly in vivo (> 1 year) (Fig. 1$).^{55}$

We hypothesized that this biodegradable nerve conduit is useful for both nerve regeneration and protective wrapping of the nerve to prevent scar and adhesion formation. In the present study, we used a rat nerve adhesion model to compare protective nerve wrapping with this nerve conduit versus hyaluronic acid (HA), which has been shown to protect nerves from adhesion formation, by examining the efficacy of each materials to prevent nerve adhesion.

\section{Methods \\ Nerve Conduit}

We used the same biodegradable polymer tube for the treatment of peripheral nerve defects as previously reported. ${ }^{22,30,55-57}$ The conduit (outer diameter $3 \mathrm{~mm}$; inner diameter $2 \mathrm{~mm}$; length $15 \mathrm{~mm}$ ) was sized so that it was 1 to $2 \mathrm{~mm}$ larger than the diameter of the rat sciatic nerves, which were less than $1 \mathrm{~mm}$, to ensure that no nerve constriction was induced..$^{25,29}$ The nerve conduit comprises an outer layer composed of a PLA multifilament fiber mesh and an inner layer composed of a 50\% PLA and 50\% PCL porous sponge with pores of 10 to $50 \mu \mathrm{m}$ (Fig. 1). This nerve conduit can maintain its tubular structure while staying very flexible. In particular, the PLA and PCL copolymer sponge of the inner layer has a honeycomb structure that is minimally damaging to the nerve. ${ }^{22,30,55-57}$

\section{Animal Model}

All experimental protocols and animal maintenance procedures were approved by the Animal Ethics Research Committee of Osaka City University Graduate School of Medicine. Lewis rats (body weight approximately $250 \mathrm{~g}$ at the time of the operation [SLC, Inc.]) were anesthetized by subcutaneous injection of $1 \mathrm{ml}$ of ketamine $(50 \mathrm{mg} /$ $\mathrm{ml}$ ) and $0.3 \mathrm{ml}$ of $2 \%$ xylazine into the dorsal back. The adhesion procedures were performed in accordance with a previously described sciatic nerve adhesion model. ${ }^{1,11,21}$ In
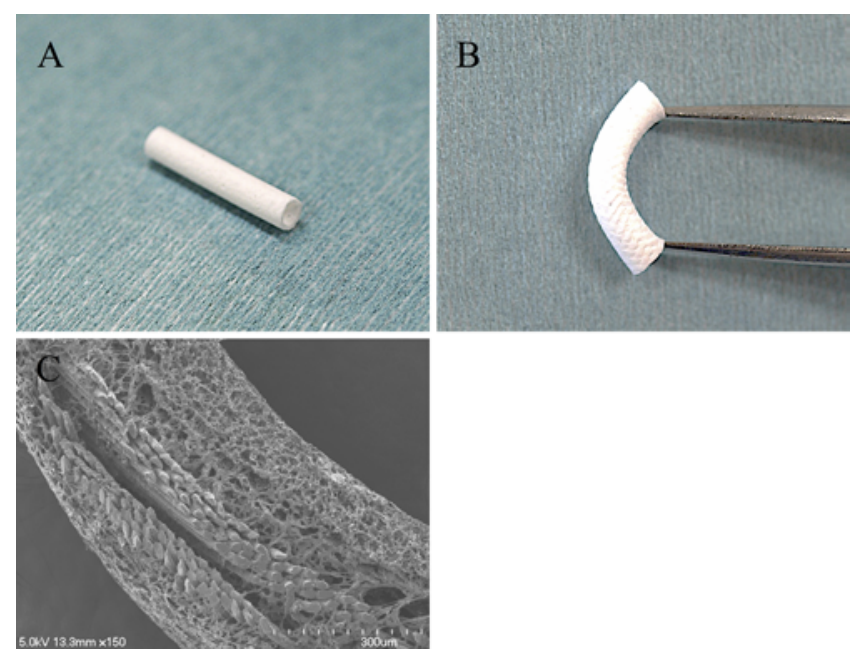

FIG. 1. The biodegradable nerve conduit. A and B: Gross appearance of the flexible nerve conduit. C: Scanning electron microscope image of a transverse section of the nerve conduit. The outer layer is composed of PLA and $50 \%$ PCA. The inner layer is a honeycomb structure containing pores.

short, the left sciatic nerve was carefully exposed and released from the surrounding tissues, including the neural bed, without injury to the nerves or vessels. Scarring and adhesion around the surgically released nerve was produced by repeatedly burning the biceps femoris muscle that composed the neural bed over a 10-mm length using a bipolar coagulator (ConMed Excalibur Plus PC; Aspen Surgical) to stimulate a local fibrotic response around the sciatic nerve.

The nerves were randomly assigned to one of the following four groups: a no-adhesion group $(n=8)$, which involved neurolysis alone without the adhesion procedure; an adhesion group $(\mathrm{n}=12)$, which involved performing the adhesion procedure after neurolysis but with no treatment; a nerve wrap group $(\mathrm{n}=12)$, which involved performing the adhesion procedure after neurolysis and subsequent wrapping with the nerve conduit; and an HA group ( $\mathrm{n}=$ 8 ), which involved performing the adhesion procedure after neurolysis and wrapping with $1 \%$ sodium HA viscous solution (Artz; Kaken). Approximately 0.5 to $1.0 \mathrm{ml}$ of HA was applied around the surgically released nerve after the adhesion procedure (Fig. 2). ${ }^{13,21}$ Six weeks after each operation, the extent of scar adhesion and the peripheral nerve function were evaluated.

\section{Gross Evaluation: Wound Healing and Adhesion Scores}

The quality of wound healing of the skin and fascia was assessed using previously described wound healing scores. ${ }^{41}$ The scores ranged from 1 to 3 (1, skin or muscle fascia entirely closed; 2 , skin or muscle fascia partially open; and 3, skin or muscle completely open). The quality of nerve adhesion was also assessed using previously described adhesion scores. ${ }^{41}$ The scores ranged from 1 to 3 (1, no dissection or mild blunt dissection; 2 , some vigorous blunt dissection required; and 3 , sharp dissection required during neurolysis to the area of adhesion). Both the wound healing score and adhesion score were calculated in each rat. 

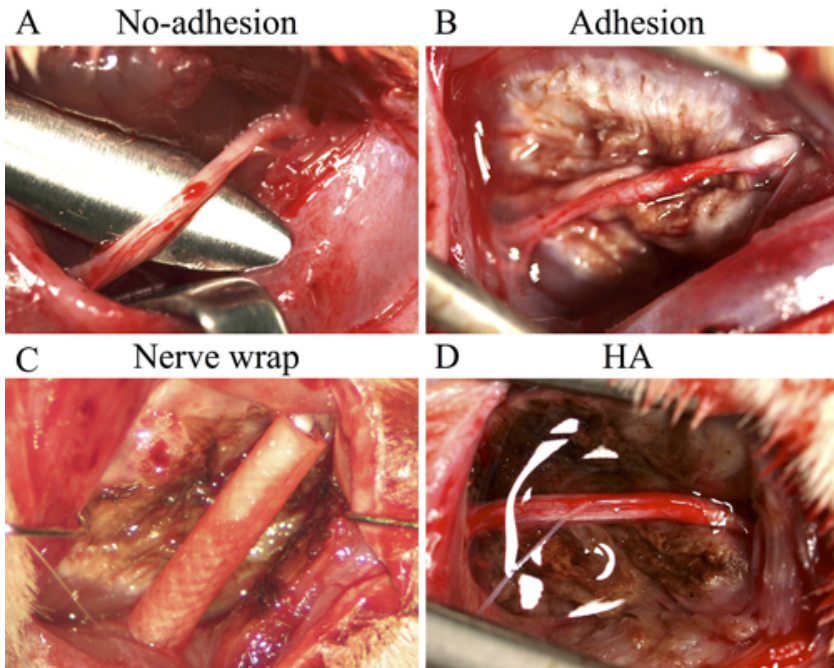

HA

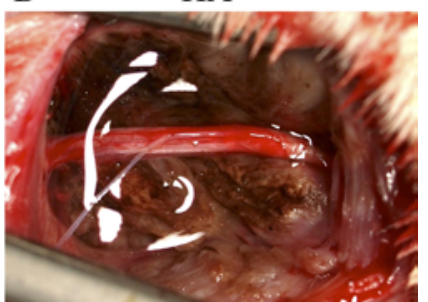

FIG. 2. Intraoperative gross findings in the experimental model. A: Noadhesion group, which involved neurolysis alone without the adhesion procedure (burning of the neural bed using a bipolar coagulator). B: Adhesion group, which involved performing the adhesion procedure after neurolysis but no subsequent treatment. C: Nerve wrap group, which involved performing the adhesion procedure after neurolysis and wrapping with the nerve conduit. D: HA group, which involved performing the adhesion procedure after neurolysis and wrapping with a $1 \%$ sodium $\mathrm{HA}$ viscous solution.

\section{Biomechanical Examination}

To evaluate the extent of adhesion, the ultimate breaking strength of the nerve that adhered to the neural bed was assessed, as previously described. ${ }^{39}$ With the operated limb fixed on the table, the nerve was ligated with 1-0 silk thread $5 \mathrm{~mm}$ proximal to the adhesion area and dissected at the proximal end. The proximal stump was then mounted on a digital force gauge (FGP-0.2; Shimpo) and subjected to traction at a rate of $2 \mathrm{~cm} / \mathrm{min}$ until the nerve was completely detached from the neural bed.

\section{Electrophysiological Examination}

To evaluate the electrophysiological function of the adherent nerve, the sciatic nerve was exposed and a bipolar stimulator was placed proximal to the adhesion area (supramaximal electrical pulses; duration $0.1 \mathrm{msec}$; frequency $1 \mathrm{~Hz}$; square wave) (VikingQuest; Natus Neurology). The compound muscle action potential of the gastrocnemius muscle was recorded by inserting the recording electrode into the central portion of the muscle. A bipolar stimulator was also placed distal to the adhesion area, 15 $\mathrm{mm}$ distal to the level of proximal stimulation, to calculate the nerve conduction velocity.

\section{Wet Weight and Histological Evaluation of Gastrocnemius Muscle}

To evaluate the functional recovery of the adherent nerve, the gastrocnemius muscles on both the affected and unaffected sides were resected and their wet weight was measured (no-adhesion group, $\mathrm{n}=6$; adhesion group, $\mathrm{n}=$ 8; nerve wrap group, $\mathrm{n}=8$; and HA group, $\mathrm{n}=6$ ). The specimens were immersed in $4 \%$ paraformaldehyde over-
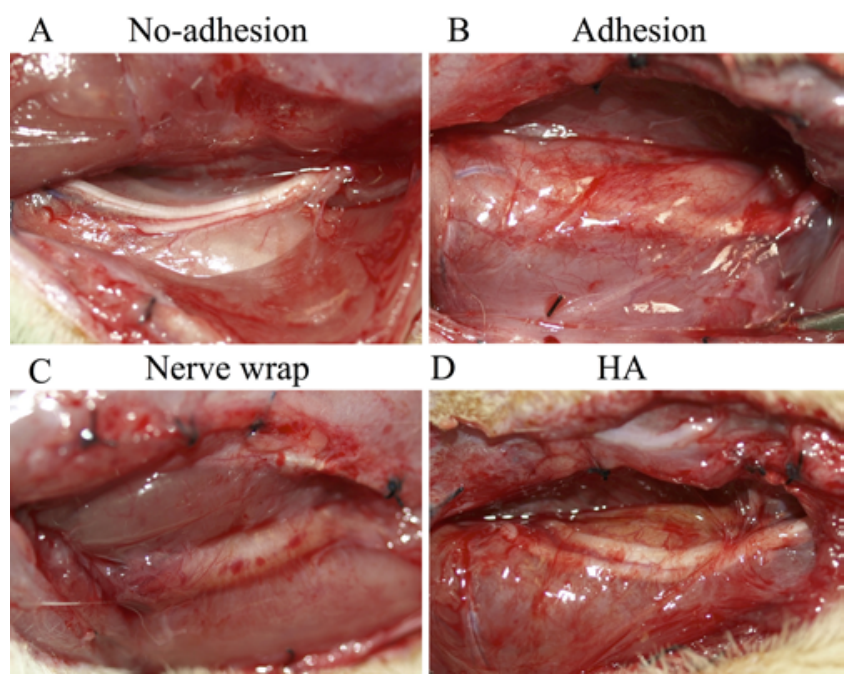

FIG. 3. Gross findings 6 weeks postoperatively. A: No-adhesion group: little scar tissue was present around the sciatic nerve. B: Adhesion group: a large amount of scar tissue covered the sciatic nerve. C: Nerve wrap group: scar tissue was present around the nerve conduit; however, the sciatic nerve was protected from scar tissue by the nerve con-

duit. D: HA group: adherent scar tissue was present between the sciatic nerve itself and the neural bed.

night and embedded in paraffin. Five-micrometer-thick transverse sections at the level of the largest area of the muscle were stained with $\mathrm{H} \& \mathrm{E}$ to evaluate the muscle atrophy.

\section{Histological Evaluation of Nerve Adhesions}

The sciatic nerve with its surrounding soft tissue, including the neural bed, was harvested. The specimens were immersed in $4 \%$ paraformaldehyde overnight and embedded in paraffin. Five-micrometer-thick central transverse sections were stained with Masson's trichrome to evaluate areas of scarring surrounding the nerve. They were also immunohistochemically stained using anti-neurofilament antibody (1:100, mouse; DAKO) to evaluate the axons. The neurofilament antibody-positive axons were morphometrically analyzed using computer-assisted imaging. An image of the transverse section of each adherent nerve was photographed at a magnification of $\times 400$ with an Olympus DP70 camera, and the number and area of the neurofilament antibody-positive axons were counted automatically using ImageJ software (National Institutes of Health) (each group, $\mathrm{n}=4$ ). To examine the inflammatory process in the areas of intraneural scar formation, the transverse sections of the nerve adhesions were also immunohistochemically stained using anti-CD68 antibody (1:500, rabbit immunoglobulin G; Abcam) for total activated macrophage markers and anti-CCR7 antibody (1:100, rabbit immunoglobulin G; Abcam) for proinflammatory M1 macrophage markers. The rats' spleen tissue was used as a positive control. ${ }^{4,7,18,22,63}$

\section{Statistical Analysis}

All data are expressed as the mean \pm standard deviation. The Mann-Whitney U-test with Bonferroni correction was used as a post hoc test after statistically signifi- 

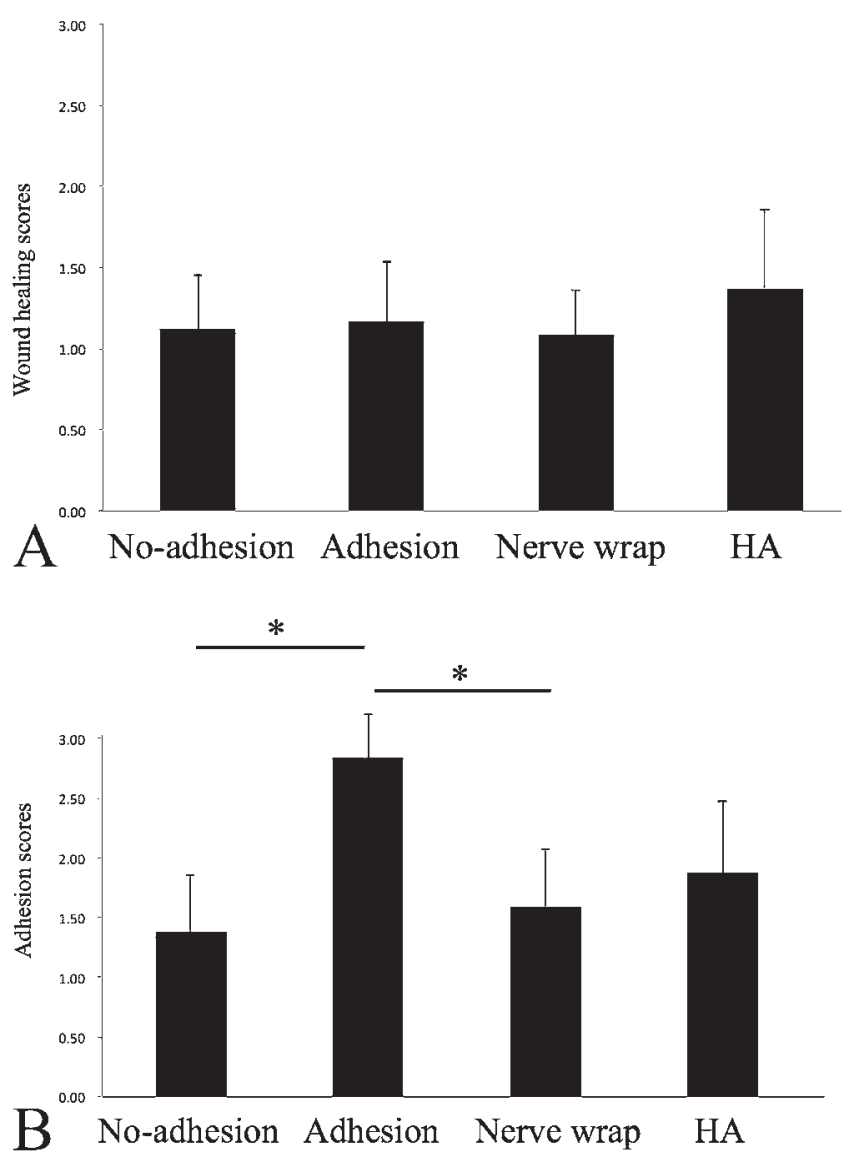

FIG. 4. Wound healing and adhesion scores 6 weeks postoperatively. A: There were no significant differences in the wound healing scores among the 4 groups. B: The adhesion scores were significantly higher in the adhesion group than in the no-adhesion and nerve wrap groups. ${ }^{*} p<0.05$.

cant differences were detected with the Kruskal-Wallis H-test, using Excel for statistical analysis. All differences were considered significant at $\mathrm{p}<0.05$.

\section{Results}

\section{Gross Evaluation (wound healing and adhesion scores)}

The gross appearance of the adherent nerves 6 weeks postoperatively is shown in Fig. 3. There were no significant differences in the wound healing scores among the 4 groups, indicating that both the superficial layer and fascia had healed without damage in all groups (Fig. 4). In contrast, the adhesion scores in the no-adhesion group and nerve wrap group were significantly lower than those in the adhesion group $(\mathrm{p}<0.05)$. The adhesion scores in the nerve wrap group were the same as those in the no-adhesion group. Although the adhesion scores in the HA group tended to be lower than those in the adhesion group, no significant differences were found between these 2 groups $(\mathrm{p}=0.41)$.

\section{Biomechanical Examination}

The ultimate breaking strength of the adherent nerves in the no-adhesion group was the lowest among the 4

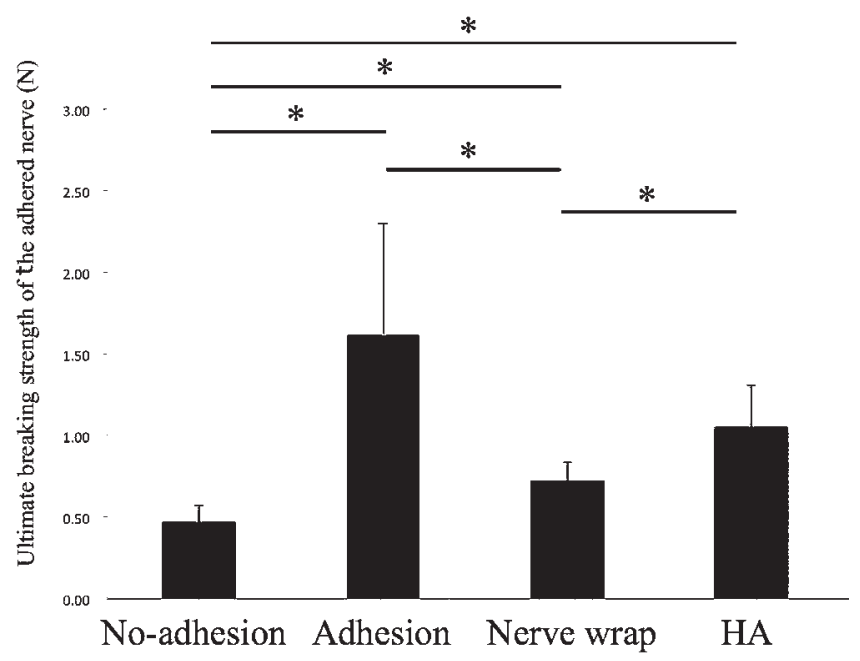

FIG. 5. The ultimate breaking strength of the adherent nerves 6 weeks postoperatively was significantly higher in the order of the adhesion, $\mathrm{HA}$, nerve wrap, and no-adhesion groups. ${ }^{*} p<0.05$.

groups, with statistical significance (adhesion group, $\mathrm{p}=$ 0.009 ; nerve wrap group, $\mathrm{p}=0.015$; and HA group, $\mathrm{p}=$ 0.024) (Fig. 5). That in the nerve wrap group was the next lowest and was significantly lower than those in the adhesion and HA groups. While the ultimate breaking strength of the adhered nerves in the HA group tended to be lower than that in the adhesion group, no significant difference was seen between these 2 groups $(p=0.41)$.

\section{Electrophysiological Examination}

The amplitude of the compound muscle action potential of the gastrocnemius muscles in the no-adhesion group was the highest among all 4 groups, with statistical significance (adhesion group, $\mathrm{p}=0.009$; nerve wrap group, $\mathrm{p}=0.025$; and HA group, $\mathrm{p}=0.024$ ) (Fig. 6). Although that in the nerve wrap group tended to be higher than that in the adhesion and HA groups, there were no significant differences among them. The motor nerve conduction velocity in the nerve wrap group was significantly higher than that in the adhesion group and the same as that in the no-adhesion group. These results indicate that nerve wrapping with the nerve conduit preserved the nerve function electrophysiologically.

\section{Wet Weight and Histological Evaluation of Gastrocnemius Muscle}

Representative images of gross appearance and histological appearance of the gastrocnemius muscle are shown in Fig. 7. The gastrocnemius muscles on the affected side atrophied in the order of the adhesion, HA, nerve wrap, and no-adhesion groups. The wet weights of the gastrocnemius muscles in the nerve wrap group were significantly greater than those in the adhesion group and HA group and the same as those in the no-adhesion group.

\section{Histological Evaluation of Adhered Nerve}

Scar tissue stained with Masson's trichrome was found around the nerve, especially between the nerve and neu- 

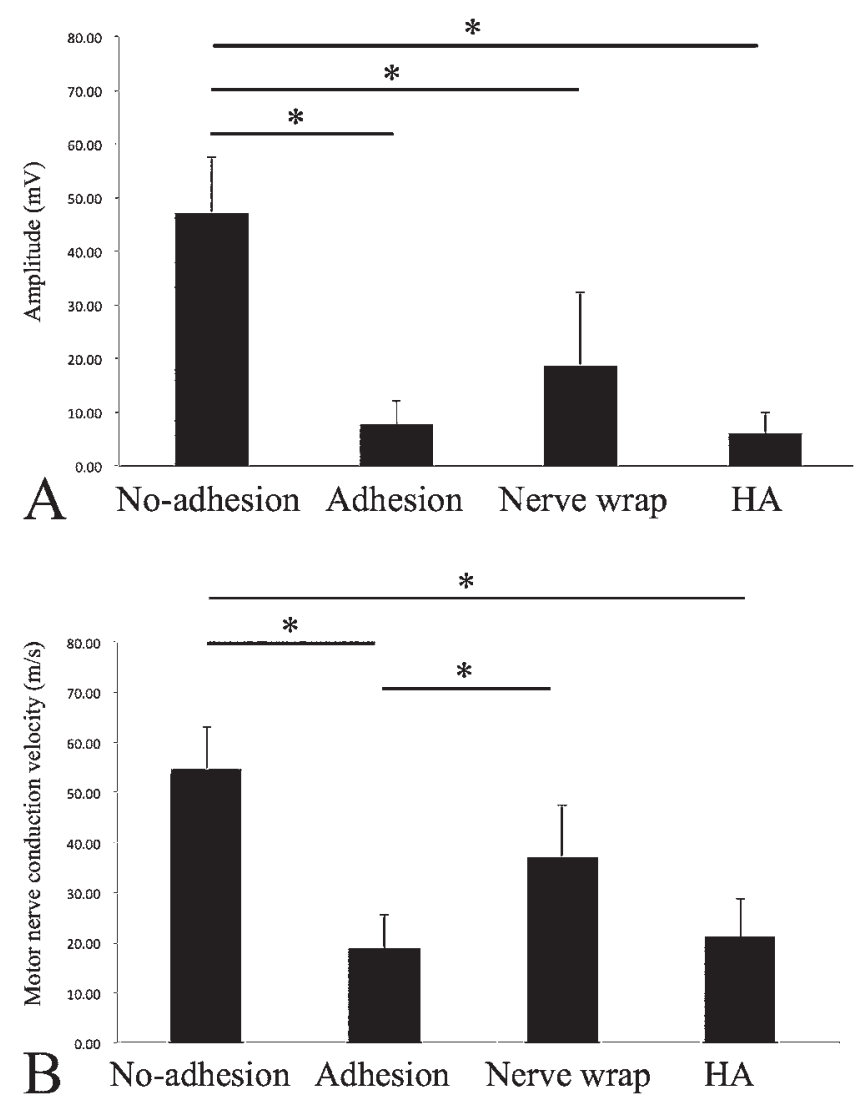

FIG. 6. Electrophysiological results. A: Amplitude of compound motor action potential of gastrocnemius muscle 6 weeks postoperatively in the adhesion group was lowest and that in the no-adhesion group was highest among all 4 groups. B: Motor nerve conduction velocity 6 weeks postoperatively in the adhesion group was lowest among all 4 groups and that in the nerve wrap group was significantly higher than that in the adhesion group. ${ }^{*} p<0.05$.

ral bed, in each group (Fig. 8). In the adhesion group, the most notable scar tissue surrounding the nerve had adhered strongly to the nerve. In contrast, a tiny amount of the scar tissue had loosely adhered to the neural bed in the no-adhesion group. In the nerve wrap group, although scar tissue was seen between the nerve conduit and neural bed, little perineural scar formation was present around the nerve because the nerve conduit had blocked it. Fibrous bridging was present between the nerve and the neural bed in the HA group, but adhesion of the nerve was looser than that in the adhesion group.

Axons in adherent nerves, which were positive for neurofilament protein, are shown in Fig. 9. The axons in the adhesion and HA groups were sparse, whereas they were packed extremely closely together and exhibited preservation of their morphological properties in the no-adhesion and nerve wrap groups. Morphometric analysis showed that the number and areas of axons that were positive for anti-neurofilament protein antibodies tended to be greater in the no-adhesion and nerve wrap groups than in the adhesion and HA groups (Fig. 9).

The highest number of CD68-positive activated macrophages was found in the intraneural scar tissue in the adhesion group, and the lowest number was found in the no-adhesion group. There were fewer CD68-positive activated macrophages in the intraneural scar tissue in the nerve wrap group than in the adhesion and HA groups. The number of CCR7-positive proinflammatory M1 macrophages was higher in the adhesion and HA groups and lower in the no-adhesion and nerve wrap groups. These findings indicate that the nerve conduits protected against macrophage invasion, especially M1 macrophage invasion (Fig. 10).

\section{Discussion}

The effect of the nerve conduit on protecting neurolysis-treated nerves from scarring and adhesion was evident in the gross appearance and in the biomechanical, electrophysiological, and histological finding of the adherent nerves in the present rat sciatic nerve adhesion model.

Once inflammation occurs in the soft tissue surrounding peripheral nerves injured by neurolysis, extraneural and intraneural scarring is established during the wound healing process, leading to adhesion of the nerve. ${ }^{48,54}$ Generally, local inflammation causes hyperpermeability of capillary vessels, migration of leukocytes including macrophages, and fibrin deposition. The activated macrophages produce transforming growth factor-beta, insulinlike growth factor, macrophage-derived growth factor, and fibroblast growth factor, allowing fibroblasts and vascular endothelial cells to migrate to the site of injury. $3,28,33,43,49$, 59,61 Adhesion of the soft tissues occurs secondary to granular and scar formation by fibroblasts and collagen fibers, wound contraction, ${ }^{17,37}$ and the replacement of connective tissues by fibrin..$^{42}$ In this proliferation phase, which lasts for several weeks, it is important for nerves to be guarded from scar tissue invasion to prevent nerve adhesion.

Many methods by which to protect peripheral nerves and prevent scar tissue formation have been devised. In the clinical setting, nerves that have undergone neurolysis may be covered by autologous vein wraps and adipose tissue or muscle flaps; however, these are associated with donor-site morbidity and a risk of surgical complications. ${ }^{12,14}$. $16,34,38,45,47,50,58,62$ Various nonbiodegradable or biodegradable materials such as silicone sheets, collagen, porcine extracellular matrix, biodegradable glass fiber wrap, PLA film, gel, and fluid have recently been used to cover neurolysis-treated nerves. ${ }^{2,3,6,9,13,15,19-21,23-26,29,31,39-41,45,46,60,64}$ NeuraGen (Integra LifeSciences Corporation), which is a nerve conduit for peripheral nerve injuries, is used as a biodegradable wrapping material for peripheral nerves and effectively induces axonal growth and protects peripheral nerves. ${ }^{2,9,31}$

The PLA-PCL material used in the present study has also been used for nerve conduits and has suitable flexibility and gentleness for nerves..$^{22,30,55-57}$ As mentioned above, it is important to protect peripheral nerves from scar formation during the initial healing stage, which continues for several weeks after neurolysis. The present study proved that the morphological properties of axons in the nerves wrapped with the nerve conduits were preserved because the PLA-PCL conduit prevented scarring and adhesion of the nerve beyond the initial healing stage without being completely absorbed and while maintaining its flexibility. 
No-adhesion
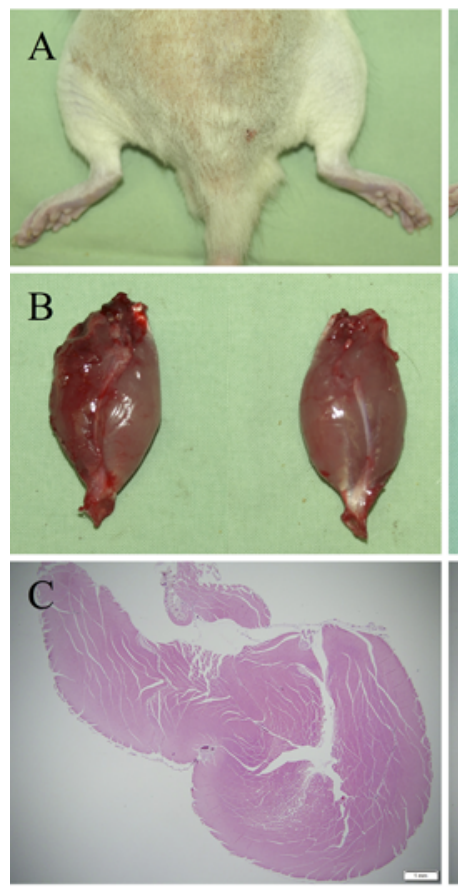

Adhesion
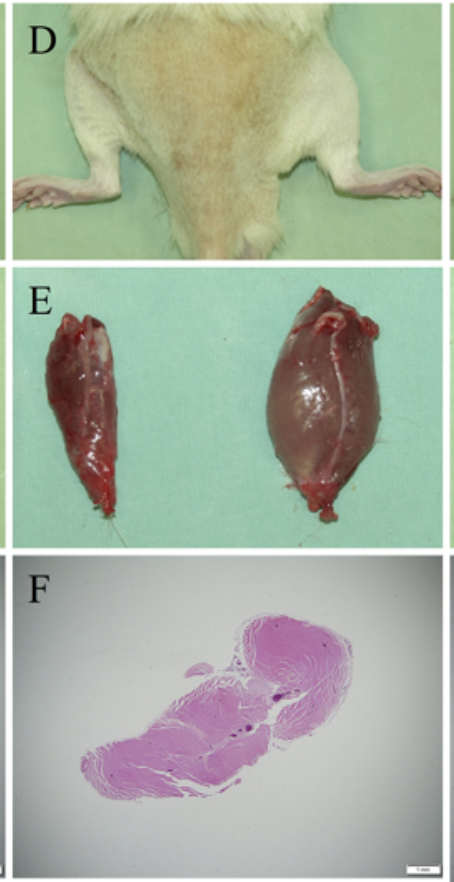
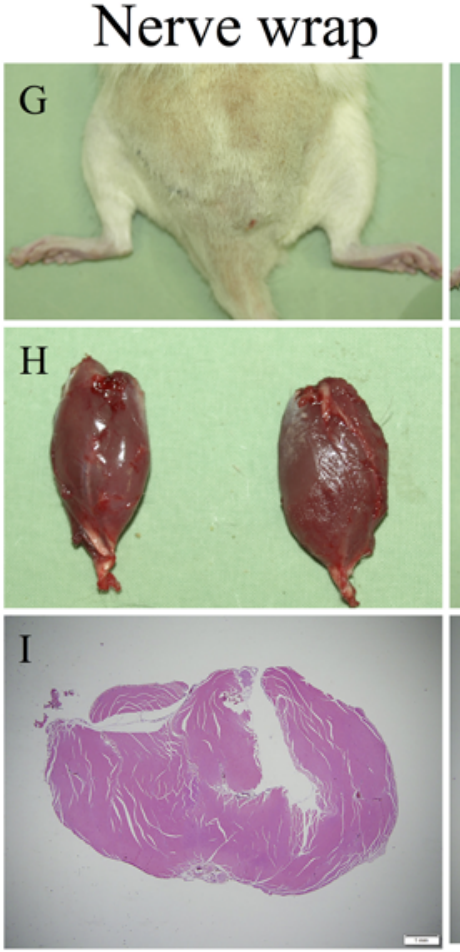
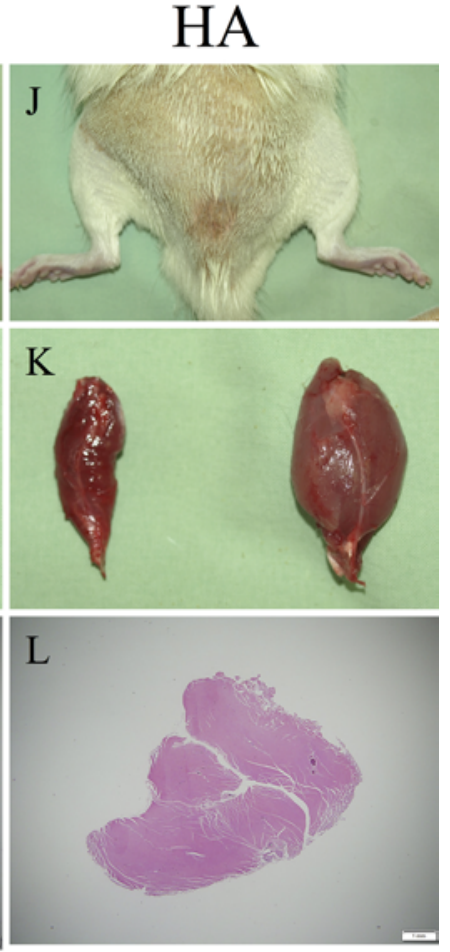

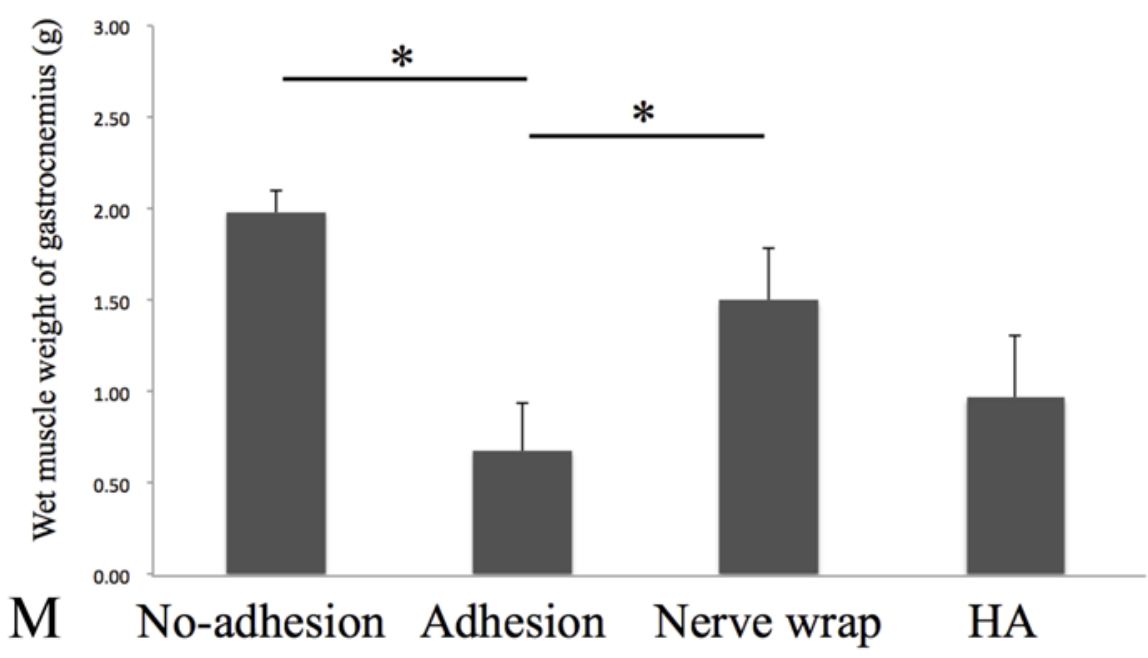

FIG. 7. Representative gross appearance of hind limbs $(\mathbf{A}, \mathbf{D}, \mathbf{G}$, and $\mathbf{J})$ and gastrocnemius muscles $(\mathrm{B}, \mathrm{E}, \mathrm{H}$, and $\mathrm{K})$ and photomicrographs (C, F, I, and L) of gastrocnemius muscle stained with $\mathrm{H} \& \mathrm{E}$ (scale bar $1 \mathrm{~mm}$ ). Wet weight of gastrocnemius muscle (M) on the affected side atrophied in the order of the adhesion, hyaluronic acid, nerve wrap, and no-adhesion groups. ${ }^{*} p<0.05$.

While HA was less effective for protection of the nerve from adhesion in the present study, we speculated that it would be absorbed earlier. PLA material for prevention of adhesions has also been recently described..$^{15,46,60}$ PLA material is hydrophobic and less adhesive to biological tissue. We previously reported that PLA material was slowly absorbed for several years without side effects. ${ }^{55}$ We believe that PLA material effectively preserves the peripheral nerves until end of scar formation.

The herein-described PLA-PCL nerve conduit can also be used as a scaffold for supportive cells and growth factors. Some growth factors, such as fibroblast growth factor, which has been shown to be effective in the prevention of scar formation, can be added to the conduit as a drug de- livery system. ${ }^{10,27,51,53,66}$ Such conduits with growth factors may provide a more effective barrier to adhesion and have greater potential for healing from the damage induced by scarring and adhesion. No reports have yet described these hybrid protective materials that contain added factors for peripheral nerve adhesion.

The mechanism of adhesion-induced peripheral nerve dysfunction remains unclear. Masear and Colgin ${ }^{34}$ have suggested that mechanical constriction of scar tissue, loss of nerve gliding and traction, and nerve ischemia cause nerve dysfunction. Murakami et al..$^{38}$ have suggested that establishment of a barrier that protects nerves, inhibition of adhesion, improvement in nerve gliding function, and the presence of several neurotrophic factors that promote 

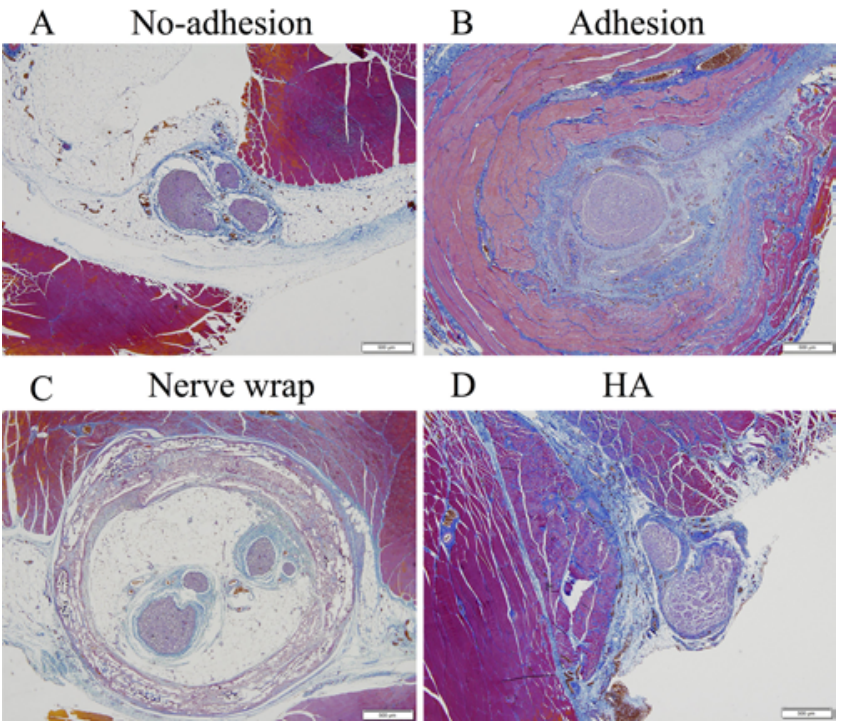

D

HA

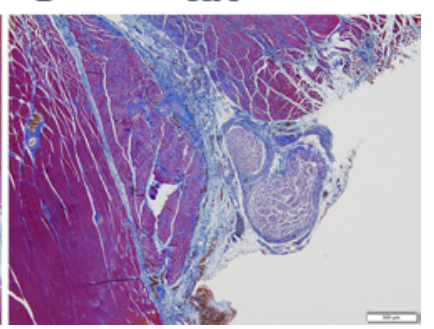

FIG. 8. Representative photomicrographs of the sciatic nerve and neural bed stained with Masson's trichrome 6 weeks postoperatively (scale bar $500 \mu \mathrm{m})$. A: Little scar tissue was present between the nerve and neural bed in the no-adhesion group. B: Notable scar tissue was surrounding the nerve and strongly adhering to the nerve in the adhesion group. C: Perineural scar formation was present in the nerve conduit in the nerve wrap group, although little scar tissue was seen between the conduit and neural bed. D: Fibrous bridging scar tissue was found between the nerve and neural bed in the HA group. axonal growth are important for prevention of adhesioninduced nerve dysfunction. Moreover, in recent years, various studies have evaluated the roles of leukocytes, mast cells, T lymphocytes, and macrophages in scar tissue formation. ${ }^{5,8,18,63}$ In the present study, nerve wrapping with the nerve conduit prevented intraneural macrophage invasion, especially inflammatory M1 macrophage invasion, and scar formation, leading to better nerve function electrophysiologically. We believe that it is important to prevent both extraneural and intraneural scar formation to maintain peripheral nerve function after neurolysis.

The main limitation in the present study is that the only evaluation time point was 6 weeks after the operation. We believe that this time point seems reasonable based on the findings of several well-supported reports. For instance, Crosio et al. ${ }^{11}$ reported that significant scar tissue-induced adhesion between the nerve and muscles had already formed 3 weeks after the same adhesion procedure as the one performed in the present study. Petersen et al ${ }^{41}$ also evaluated a sciatic nerve adhesion model and reported that a substantial degree of scar formation had been established earlier than 6 weeks postoperatively. Ohsumi et al. ${ }^{39}$ showed that the blood-nerve barrier had recovered by 6 weeks after neurolysis and provided only a single time point (6 weeks postoperatively) for evaluation of sciatic nerve adhesion. Further studies are needed to evaluate the long-term effects of the nerve conduit because this PLAPCL material is not only biodegradable but also artificial, and there is a possibility of side effects that interfere with the biological tissues.
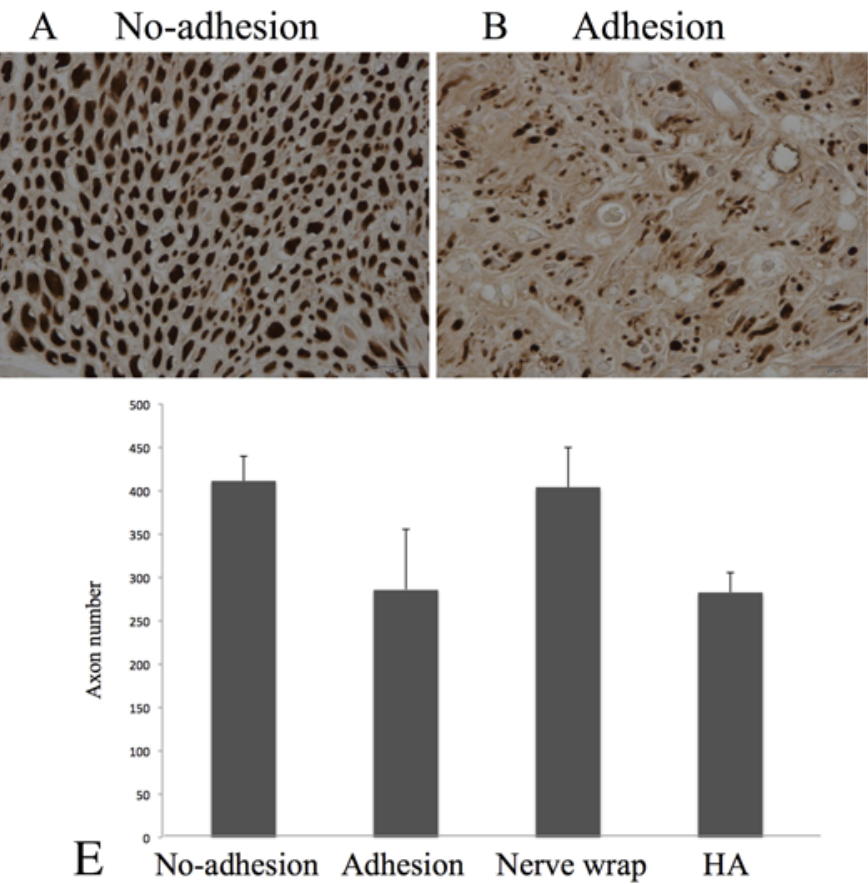

C Nerve wrap
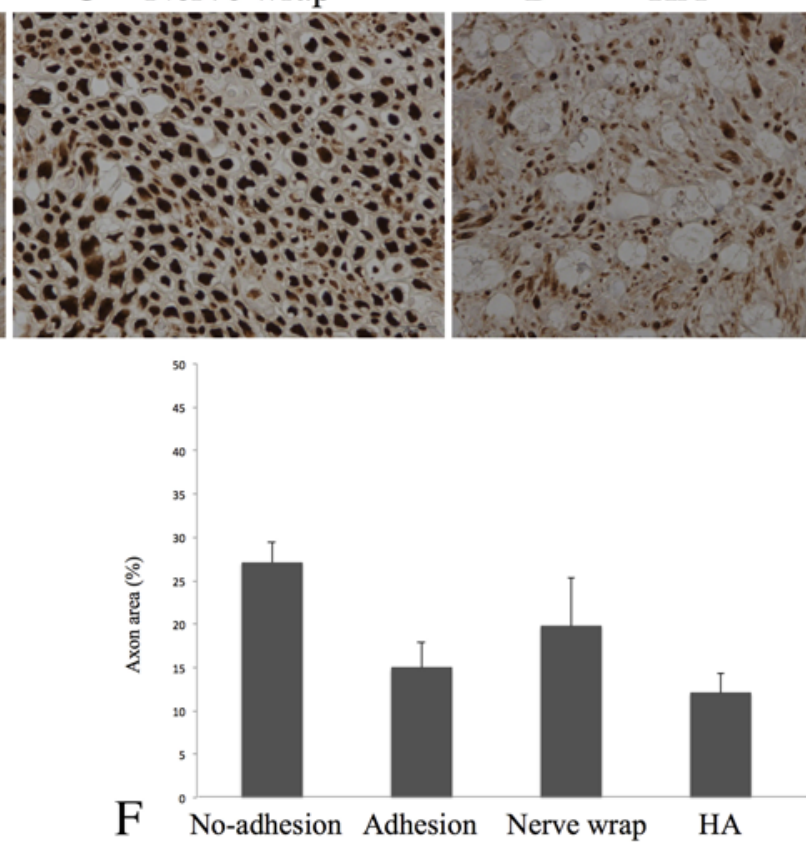

FIG. 9. Representative photomicrographs of axons stained using anti-neurofilament antibody 6 weeks postoperatively (scale bar $20 \mu \mathrm{m})$. A: The morphological properties of the axons were preserved in the no-adhesion group. B: Numerous but sparse smaller-diameter axons were seen in the adhesion group. C: The morphological properties of the axons were relatively well preserved in the nerve wrap group, although the diameter of the axons was smaller than that it was in the no-adhesion group. D: Numerous but sparse smaller-diameter axons were seen in the HA group, as in the adhesion group. $\mathbf{E}$ and F: The axon numbers and areas of neurofilament protein-positive axons were higher in the no-adhesion and nerve wrap groups than in the adhesion and $\mathrm{HA}$ groups 6 weeks postoperatively. ${ }^{*} p<0.05$. 


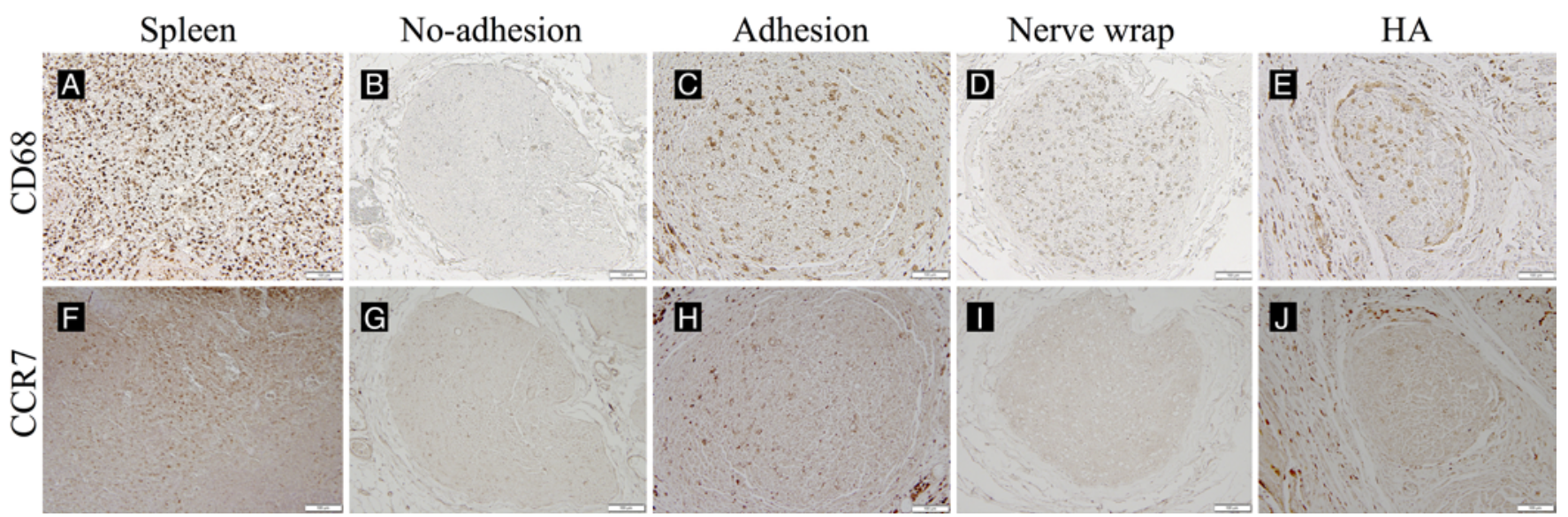

FIG. 10. Representative histological photomicrographs of intraneural scar tissue stained with anti-CD68 antibody and anti-CCR7 antibody 6 weeks postoperatively (scale bar $100 \mu \mathrm{m}$ ). The highest numbers of CD68-positive activated macrophages and CCR7positive proinflammatory M1 macrophages were found in the intraneural scar tissue in the adhesion group, whereas the lowest numbers were found in the no-adhesion group. There were fewer CD68-positive activated macrophages and CCR7-positive proinflammatory M1 macrophages in the intraneural scar tissue in the nerve wrap group than there were in the adhesion and HA groups.

\section{Conclusions}

This PLA-PCL nerve conduit protected peripheral nerves that underwent neurolysis from the development of adhesion and preserved the nerve function in a rat nerve adhesion model. The nerve conduit effectively blocked scarring and prevented adhesion-related damage in the peripheral nerves.

\section{References}

1. Abe Y, Doi K, Sakai K, Yamamoto H, Kawai S: Experimental study of the peripheral nerve excursion. J Jpn Soc Surg Hand 11:480-484, 1994 (Jpn)

2. Archibald SJ, Shefner J, Krarup C, Madison RD: Monkey median nerve repaired by nerve graft or collagen nerve guide tube. J Neurosci 15:4109-4123, 1995

3. AxoGen: AxoGuard ${ }^{\circledR}$ NerveProtector. (http://www. axogeninc.com/products/4144/axoguard-nerve-protector) [Accessed June 6, 2017]

4. Baum CL, Arpey CJ: Normal cutaneous wound healing: clinical correlation with cellular and molecular events. Dermatol Surg 31:674-686, 2005

5. Binnebösel M, Rosch R, Junge K, Lynen-Jansen P, Schumpelick V, Klinge U: Macrophage and T-lymphocyte infiltrates in human peritoneal adhesions indicate a chronic inflammatory disease. World J Surg 32:296-304, 2008

6. Braga-Silva J: The use of silicone tubing in the late repair of the median and ulnar nerves in the forearm. J Hand Surg [Br] 24:703-706, 1999

7. Brown BA: Internal neurolysis in traumatic peripheral nerve lesions in continuity. Surg Clin North Am 52:1167-1175, 1972

8. Burnett SH, Beus BJ, Avdiushko R, Qualls J, Kaplan AM, Cohen DA: Development of peritoneal adhesions in macrophage depleted mice. J Surg Res 131:296-301, 2006

9. Bush MD, Li Z, Koman LA: Surgical management of recurrent carpal tunnel syndrome. Tech Orthop 21:54-60, 2006

10. Choi W, Kawanabe H, Sawa Y, Taniguchi K, Ishikawa H: Effects of bFGF on suppression of collagen type I accumulation and scar tissue formation during wound healing after mucoperiosteal denudation of rat palate. Acta Odontol Scand 66:31-37, 2008

11. Crosio A, Valdatta L, Cherubino M, Izzo M, Pellegatta I,
Pascal D, et al: A simple and reliable method to perform biomechanical evaluation of postoperative nerve adhesions. J Neurosci Methods 233:73-77, 2014

12. Dahlin LB, Lekholm C, Kardum P, Holmberg J: Coverage of the median nerve with free and pedicled flaps for the treatment of recurrent severe carpal tunnel syndrome. Scand J Plast Reconstr Surg Hand Surg 36:172-176, 2002

13. Dam-Hieu P, Lacroix C, Said G, Devanz P, Liu S, Tadie M: Reduction of postoperative perineural adhesions by Hyaloglide gel: an experimental study in the rat sciatic nerve. Neurosurgery 56 (2 Suppl):425-433, 2005

14. Dumanian GA, McClinton MA, Brushart TM: The effects of free fat grafts on the stiffness of the rat sciatic nerve and perineural scar. J Hand Surg Am 24:30-36, 1999

15. Fukuhira Y, Ito M, Kaneko H, Sumi Y, Tanaka M, Yamamoto $\mathrm{S}$, et al: Prevention of postoperative adhesions by a novel honeycomb-patterned poly(lactide) film in a rat experimental model. J Biomed Mater Res B Appl Biomater 86:353-359, 2008

16. Fusetti C, Garavaglia G, Mathoulin C, Petri JG, Lucchina $\mathrm{S}$ : A reliable and simple solution for recalcitrant carpal tunnel syndrome: the hypothenar fat pad flap. Am J Orthop 38:181-186, 2009

17. Gabbiani G, Ryan GB, Majne G: Presence of modified fibroblasts in granulation tissue and their possible role in wound contraction. Experientia 27:549-550, 1971

18. Gordon S, Martinez FO: Alternative activation of macrophages: mechanism and functions. Immunity 32:593-604, 2010

19. Hodde J, Janis A, Ernst D, Zopf D, Sherman D, Johnson C: Effects of sterilization on an extracellular matrix scaffold: part I. Composition and matrix architecture. J Mater Sci Mater Med 18:537-543, 2007

20. Hodde J, Janis A, Hiles M: Effects of sterilization on an extracellular matrix scaffold: part II. Bioactivity and matrix interaction. J Mater Sci Mater Med 18:545-550, 2007

21. Ikeda K, Yamauchi D, Osamura N, Hagiwara N, Tomita K: Hyaluronic acid prevents peripheral nerve adhesion. Br J Plast Surg 56:342-347, 2003

22. Ikeda M, Uemura T, Takamatsu K, Okada M, Kazuki K, Tabata $Y$, et al: Acceleration of peripheral nerve regeneration using nerve conduits in combination with induced pluripotent stem cell technology and a basic fibroblast growth factor drug delivery system. J Biomed Mater Res A 102:1370-1378, 2014 
23. Jeans LA, Gilchrist T, Healy D: Peripheral nerve repair by means of a flexible biodegradable glass fibre wrap: a comparison with microsurgical epineurial repair. J Plast Reconstr Aesthet Surg 60:1302-1308, 2007

24. Kehoe S, Zhang XF, Boyd D: FDA approved guidance conduits and wraps for peripheral nerve injury: a review of materials and efficacy. Injury 43:553-572, 2012

25. Kim PD, Hayes A, Amin F, Akelina Y, Hays AP, Rosenwasser MP: Collagen nerve protector in rat sciatic nerve repair: A morphometric and histological analysis. Microsurgery 30:392-396, 2010

26. Kline DG, Hayes GJ: The use of a resorbable wrapper for peripheral-nerve repair; experimental studies in chimpanzees. J Neurosurg 21:737-750, 1964

27. Kobayashi T, Mizuta M, Hiwatashi N, Kishimoto Y, Nakamura T, Kanemaru SI, et al: Drug delivery system of basic fibroblast growth factor using gelatin hydrogel for restoration of acute vocal fold scar. Auris Nasus Larynx 44:86-92, 2017

28. Koh TJ, DiPietro LA: Inflammation and wound healing: the role of the macrophage. Expert Rev Mol Med 13:e23, 2011

29. Kokkalis ZT, Pu C, Small GA, Weiser RW, Venouziou AI, Sotereanos DG: Assessment of processed porcine extracellular matrix as a protective barrier in a rabbit nerve wrap model. J Reconstr Microsurg 27:19-28, 2011

30. Koshimune M, Takamatsu K, Nakatsuka H, Inui K, Yamano Y, Ikada Y: Creating bioabsorbable Schwann cell coated conduits through tissue engineering. Biomed Mater Eng 13:223-229, 2003

31. Li ST, Archibald SJ, Krarup C, Madison RD: Peripheral nerve repair with collagen conduits. Clin Mater 9:195-200, 1992

32. Lundborg G, Rydevik B: Effects of stretching the tibial nerve of the rabbit. A preliminary study of the intraneural circulation and the barrier function of the perineurium. J Bone Joint Surg Br 55:390-401, 1973

33. Martin P: Wound healing-aiming for perfect skin regeneration. Science 276:75-81, 1997

34. Masear VR, Colgin S: The treatment of epineural scarring with allograft vein wrapping. Hand Clin 12:773-779, 1996

35. McCall TD, Grant GA, Britz GW, Goodkin R, Kliot M: Treatment of recurrent peripheral nerve entrapment problems: role of scar formation and its possible treatment. Neurosurg Clin N Am 12:329-339, 2001

36. Millesi H, Zöch G, Rath T: The gliding apparatus of peripheral nerve and its clinical significance. Ann Chir Main Memb Super 9:87-97, 1990

37. Montandon D, D'andiran G, Gabbiani G: The mechanism of wound contraction and epithelialization: clinical and experimental studies. Clin Plast Surg 4:325-346, 1977

38. Murakami K, Kuniyoshi K, Iwakura N, Matsuura Y, Suzuki T, Takahashi K, et al: Vein wrapping for chronic nerve constriction injury in a rat model: study showing increases in VEGF and HGF production and prevention of pain-associated behaviors and nerve damage. J Bone Joint Surg Am 96:859-867, 2014

39. Ohsumi H, Hirata H, Nagakura T, Tsujii M, Sugimoto T, Miyamoto K, et al: Enhancement of perineurial repair and inhibition of nerve adhesion by viscous injectable pure alginate sol. Plast Reconstr Surg 116:823-830, 2005

40. Okui N, Yamamoto M, Fukuhira Y, Kaneko H, Hirata H: A new nerve coaptation technique using a biodegradable honeycomb-patterned film. Microsurgery 32:466-474, 2012

41. Petersen J, Russell L, Andrus K, MacKinnon M, Silver J, Kliot M: Reduction of extraneural scarring by ADCON-T/N after surgical intervention. Neurosurgery 38:976-984, 1996

42. Prockop DJ, Kivirikko KI, Tuderman L, Guzman NA: The biosynthesis of collagen and its disorders (first of two parts). N Engl J Med 301:13-23, 1979

43. Pull SL, Doherty JM, Mills JC, Gordon JI, Stappenbeck TS: Activated macrophages are an adaptive element of the colonic epithelial progenitor niche necessary for regenerative responses to injury. Proc Natl Acad Sci U S A 102:99-104, 2005

44. Rydevik B, Lundborg G, Nordborg C: Intraneural tissue reactions induced by internal neurolysis. An experimental study on the blood-nerve barrier, connective tissues and nerve fibres of rabbit tibial nerve. Scand J Plast Reconstr Surg 10:3-8, 1976

45. Siemionow M, Uygur S, Ozturk C, Siemionow K: Techniques and materials for enhancement of peripheral nerve regeneration: a literature review. Microsurgery 33:318-328, 2013

46. Simamora P, Chern W: Poly-L-lactic acid: an overview. J Drugs Dermatol 5:436-440, 2006

47. Sotereanos DG, Giannakopoulos PN, Mitsionis GI, Xu J, Herndon JH: Vein-graft wrapping for the treatment of recurrent compression of the median nerve. Microsurgery 16:752-756, 1995

48. Stadelmann WK, Digenis AG, Tobin GR: Physiology and healing dynamics of chronic cutaneous wounds. Am J Surg 176 (2A Suppl):26S-38S, 1998

49. Steed DL: The role of growth factors in wound healing. Surg Clin North Am 77:575-586, 1997

50. Strickland JW, Idler RS, Lourie GM, Plancher KD: The hypothenar fat pad flap for management of recalcitrant carpal tunnel syndrome. J Hand Surg Am 21:840-848, 1996

51. Suehiro A, Hirano S, Kishimoto Y, Rousseau B, Nakamura T, Ito J: Treatment of acute vocal fold scar with local injection of basic fibroblast growth factor: a canine study. Acta Otolaryngol 130:844-850, 2010

52. Sunderland S: The nerve lesion in the carpal tunnel syndrome. J Neurol Neurosurg Psychiatry 39:615-626, 1976

53. Tateya I, Tateya T, Sohn JH, Bless DM: Histological effect of basic fibroblast growth factor on chronic vocal fold scarring in a rat model. Clin Exp Otorhinolaryngol 9:56-61, 2016

54. Tobin GR: Wound repair: biologic foundations and clinical considerations, in Richardson JD, Polk HC Jr, Flint LM Jr (eds): Trauma: Clinical Care and Pathophysiology. Chicago: Year Book Medical Publishers, 1987, pp 213-261

55. Uemura T, Ikeda M, Takamatsu K, Yokoi T, Okada M, Nakamura H: Long-term efficacy and safety outcomes of transplantation of induced pluripotent stem cell-derived neurospheres with bioabsorbable nerve conduits for peripheral nerve regeneration in mice. Cells Tissues Organs 200:78-91, 2014

56. Uemura T, Takamatsu K, Ikeda M, Okada M, Kazuki K, Ikada Y, et al: A tissue-engineered bioabsorbable nerve conduit created by three-dimensional culture of induced pluripotent stem cell-derived neurospheres. Biomed Mater Eng 21:333339,2011

57. Uemura T, Takamatsu K, Ikeda M, Okada M, Kazuki K, Ikada Y, et al: Transplantation of induced pluripotent stem cellderived neurospheres for peripheral nerve repair. Biochem Biophys Res Commun 419:130-135, 2012

58. Uemura T, Takamatsu K, Okada M, Ikeda M, Nakamura $\mathrm{H}$ : Radial artery perforator adiposal flap for coverage of the scarred median nerve. J Plast Reconstr Aesthet Surg 66:1019-1021, 2013

59. Wahl SM, Wahl LM, McCarthy JB: Lymphocyte-mediated activation of fibroblast proliferation and collagen production. J Immunol 121:942-946, 1978

60. Welch WC, Cornwall GB, Toth JM, Turner AS, Thomas KA, Gerszten PC, et al: Use of polylactide resorbable film as an adhesion barrier. Orthopedics 25 (10 Suppl):s1121-s1130, 2002

61. Werner S, Grose R: Regulation of wound healing by growth factors and cytokines. Physiol Rev 83:835-870, 2003

62. Xu J, Sotereanos DG, Moller AR, Jacobsohn J, Tomaino MM, Fischer KJ, et al: Nerve wrapping with vein grafts in a rat model: a safe technique for the treatment of recurrent chronic compressive neuropathy. J Reconstr Microsurg 14:323-330, 1998 
63. Xu X, Rivkind A, Pappo O, Pikarsky A, Levi-Schaffer F: Role of mast cells and myofibroblasts in human peritoneal adhesion formation. Ann Surg 236:593-601, 2002

64. Yamamoto M, Endo N, Ito M, Okui N, Koh S, Kaneko H, et al: Novel polysaccharide-derived hydrogel prevents perineural adhesions in a rat model of sciatic nerve adhesion. $\mathbf{J}$ Orthop Res 28:284-288, 2010

65. Yamamoto M, Okui N, Tatebe M, Shinohara T, Hirata H: Regeneration of the perineurium after microsurgical resection examined with immunolabeling for tenascin- $\mathrm{C}$ and alpha smooth muscle actin. J Anat 218:413-425, 2011

66. Yuan Z, Zhao J, Chen Y, Yang Z, Cui W, Zheng Q: Regulating inflammation using acid-responsive electrospun fibrous scaffolds for skin scarless healing. Mediators Inflamm 2014:858045, 2014

\section{Disclosures}

The authors report no conflict of interest concerning the materi- als or methods used in this study or the findings specified in this paper.

\section{Author Contributions}

Conception and design: Shintani, Uemura, Takamatsu, Nakamura. Acquisition of data: Shintani, Uemura, Yokoi, Onode. Analysis and interpretation of data: Shintani, Uemura. Drafting the article: Shintani, Uemura. Critically revising the article: Shintani, Uemura. Reviewed submitted version of manuscript: Shintani, Uemura. Approved the final version of the manuscript on behalf of all authors: Shintani. Statistical analysis: Shintani. Administrative/technical/material support: Shintani, Uemura, Yokoi, Onode. Study supervision: Shintani, Uemura, Takamatsu, Okada, Nakamura.

\section{Correspondence}

Kosuke Shintani, Department of Orthopaedic Surgery, Osaka City University Graduate School of Medicine, 1-4-3 Asahimachi Abeno-ku, Osaka 545-8585, Japan. email: qq355dzt9@msic.med. osaka-cu.ac.jp. 